\title{
Phase Wettability and Microstructural Evolution in Solid Oxide Fuel Cell Anode Materials
}

\author{
Ryan Davis $^{\mathrm{a}}$, Fadi Abdeljawad ${ }^{\mathrm{a}}$, Jeffrey Lillibridge ${ }^{\mathrm{b}}$, Mikko Haataja ${ }^{\mathrm{a}, \mathrm{c}, \mathrm{d}, *}$ \\ ${ }^{a}$ Department of Mechanical and Aerospace Engineering, Princeton University, Princeton, \\ NJ 08544, USA \\ ${ }^{b}$ Department of Physics, California State University, Northridge, CA 91330, USA \\ ${ }^{c}$ Princeton Institute for the Science and Technology of Materials (PRISM), Princeton \\ University, Princeton, NJ 08544, USA \\ ${ }^{d}$ Program in Applied and Computational Mathematics (PACM), Princeton University, \\ Princeton, NJ 08544, USA
}

\begin{abstract}
Recent experimental and theoretical findings suggest that high temperature solid oxide fuel cells (SOFCs) often suffer from performance degradation due to coarsening of the metallic phase particles within the anode. In this study, we explore the feasibility of improving the microstructural stability of SOFC anode materials by tuning the contact angle between the metallic phase and electrolyte particles. To this end, a continuum diffuse-interface model is employed to capture the coarsening behavior of the metallic phase and simulate a range of equilibrium contact angles. The evolution of performance-critical, microstructural features is presented for varying degrees of phase wettability. It is found that both the density of electrochemically active triple phase regions and contiguity of the electron-conducting phase display undesirable minima near the contact angle of conventional SOFC materials. Our results suggest that tailoring the interfacial properties of the constituent phases could lead to a significant increase in the performance and lifetime of SOFCs.
\end{abstract}

Keywords: solid oxide fuel cell, microstructure, coarsening, modeling

*Corresponding author. Tel.: 1-609-2589126; fax: 1-609-2585877.

Preprint submitted to Elsevier

June 16, 2014

(C) 2014. This manuscript version is made available under the Elsevier user license http://www.elsevier.com/open-access/userlicense/1.0/ 


\section{Introduction}

Solid oxide fuel cells (SOFCs) are devices that convert chemical energy directly to electrical energy. They consist of three components: the cathode where oxygen gas is reduced to form ions, the electrolyte which mediates ion diffusion, and the anode where the oxygen ions react with fuel to produce electricity, water, and other by-products. Conventional SOFCs utilize a thin layer of yttria-stabilized zirconia (YSZ) as the ceramic electrolyte and a porous, heterogeneous mixture of nickel (Ni) and YSZ for a cermet anode [1]. The widespread adoption of this design is due to its numerous advantages including high conversion efficiencies, which reach up to $60 \%$ for stand-alone fuel cells and up to $80 \%$ with co-generation [2]. Additionally, SOFCs benefit from the ability to directly utilize hydrocarbon fuels through internal reforming and exhibit greatly reduced carbon emissions compared to internal combustion [3].

Although Ni-YSZ SOFCs are a promising technology, they suffer from many drawbacks that lead to gradual performance loss over the course of the operation lifetime [4]. A specific degradation mechanism, which has been observed as a major contributor to performance loss, is the agglomeration and coarsening of Ni particles in the anode $[5,6,7]$. Due to the high operating temperatures of SOFCs $\left(800-1000^{\circ} \mathrm{C}\right)$ required for sufficient ion diffusion through the YSZ electrolyte, the nickel phase coarsens, which reduces both the amount of available reaction sites (triple-phase boundary lines) and contiguous paths for electron transport [8]. Coarsening within a heterogeneous cermet along with a multitude of fabrication microstructures make designing robust anodes challenging. These issues must be resolved for SOFC technology to reach the reliability required for widespread commercial use.

In an effort to overcome the difficulties associated with the Ni-YSZ SOFC design, there has been continued exploration of alternative materials that may provide improved performance [9]. These alterations include doping $\mathrm{Ni}$ with other refractory metals, employing metal alloys, and substituting nickel entirely with other electrically conductive materials [10, 11]. Employing alternative materials affects changes in both the electrochemical properties and microstructural features of the anode. For example, a change in the materials used within the SOFC anode brings about a concomitant change in interfacial properties $[12,13]$, and, correspondingly, the potential to increase cell performance. In particular, experimental findings by Ringuedé et al. [14] suggest that alloying nickel with various amounts of cobalt results in 
an extension of the triple phase boundary and a significant increase in fuel cell performance. Additionally, Skarmoutsos et al. [15] have shown that by doping YSZ with titanium, dramatic losses in performance experienced by an SOFC during initial operation can be avoided.

Despite considerable experimental evidence showing performance increases using variations on the Ni-YSZ materials selection, there has not been a thorough parametric investigation into this matter. To this end, herein we employ a continuum diffuse-interface (or phase field) method [16] to model the evolution of the nickel phase within the heterogeneous Ni-YSZ cermet anode. The phase field method is particularly well suited for this problem due to its ability to naturally treat singular reconfiguration events occuring during the microstructural evolution process, such as the merging and splitting of domains. Multiple order parameters can be employed to model the multiple phases of the cermet. Additionally, use of the phase field method at the mesoscale affords the spatial resolution required to capture the heterogeneous nature of the anode while still accessing the time scales necessary to observe long-term, degradation mechanisms [7]. Previous computational studies of SOFC anodes using the phase field method have displayed results in accordance with experimental observations [17, 18, 19, 20].

In this parametric study, to assess the potential efficacy of tuning the interfacial energetics of the system to achieve improved microstructural characteristics, the equilibrium contact angle between the metallic and ceramic phases is systematically varied over a broad range, incorporating both poorly and complete wetting regimes. For each contact angle, several anode systems, represented as random closed packed (RCP) structures of spherical particles, are numerically evolved to yield coarsened metallic phase microstructures. These microstructures are subsequently analyzed for performance critical features such as average metallic phase diameter, triple-phase boundary density, and contiguity of the electron conducting phase. It is found that both the density of electrochemically active triple phase regions and contiguity of the electron-conducting phase display undesirable minima near the contact angle of conventional SOFC materials. Our results suggest that tailoring the interfacial properties of the constituent phases could lead to significant increases in the performance and lifetime of SOFCs.

The rest of this manuscript is organized as follows. In Sec. 2, the diffuseinterface modeling framework is outlined, followed by a discussion of microstructural characterization techniques employed in the analysis of the simulated morphologies in Sec. 3. Simulation results are then analyzed and 
discussed in Sec. 4. Finally, concluding remarks are presented in Sec. 5.

\section{Diffuse-interface Formalism}

As usual, the starting point of our diffuse-interface approach is the introduction of order parameters (OPs) that describe each phase in porous SOFC cermets $[17,18,19,20]$. Next, a thermodynamic free energy $\mathcal{F}_{\text {tot }}$ is constructed in terms of the OPs, whose spatio-temporal evolution follows from the minimization of $\mathcal{F}_{\text {tot }}$. Motivated by experimental findings on YSZ suggesting that the ceramic phase is virtually stationary relative to the metallic one $[5,17]$, a static, non-evolving, field $\phi(\mathbf{r})$ is assigned to the ceramic phase to reflect its microstructural stability. On the other hand, a field $c(\mathbf{r}, t)$ is used to track the evolution of the metallic phase as its coarsens in SOFC anodes under ambient conditions. As in standard phase field methods, OPs take on constant values within the bulk phases, but vary smoothly and continuously across interfaces. In this work, the equilibrium values of the OPs within the bulk phases are chosen such that in the (a) metallic phase $(\phi, c)=(0,+1)$, (b) ceramic one $(\phi, c)=(+1,-1)$, and (c) pore phase $(\phi, c)=(0,-1)$. Additionally, metallic and ceramic interfaces are defined via $c(\mathbf{r}, t) \in(-1,1)$ and $\phi(\mathbf{r}) \in(0,1)$, respectively.

As in standard Ginzburg-Landau formalism and utilizing the single crystal formalism of our recently developed model for SOFC anode systems [7], the total chemical free energy is written as

$$
\mathcal{F}_{\text {tot }}=\int d \mathbf{r} f_{\text {tot }}=\int d \mathbf{r}\left[f_{\text {hom }}(c)+\frac{W_{c}^{2}}{2}|\nabla c|^{2}+f_{\text {wett }}(c, \phi)\right],
$$

where $f_{\text {hom }}(c)=A_{c}\left(c^{2}-1\right)^{2}$ is a fourth-order Landau polynomial for the homogeneous free energy of the metallic phase, and the parameter $A_{c}$ sets the energy scale. The gradient energy coefficient, $W_{c}$, together with $A_{c}$, determine the interfacial energy of the metallic phase. The last term on the right hand side of Eq. (1) is an energy density term that accounts for the interaction between the metallic and ceramic phases and controls the resulting equilibrium wetting angle. In this work, $f_{\text {wett }}(c, \phi)$ is written as

$$
f_{w e t t}(c, \phi)=-\xi_{1} c|\nabla \phi|^{2}+\xi_{2} \phi\left(c-\frac{c^{3}}{3}\right)
$$


where the first term on the right hand side encourages the metallic and ceramic phases to share a common interface, with $\xi_{1}$ a tunable model parameter. Concurrently, the second term on the right hand side of Eq. (2) is an obstacle potential that penalizes spatial overlap of the metal and ceramic phases. The interplay of these two coupling terms is employed to control the wettability of the two phases and set the desired equilibrium contact angle $\theta_{c}$.

With the proposed total chemical free energy functional in Eq. (1) and following the treatment of Cahn and Hilliard [21], the metallic phase interface energy and width in an SOFC anode can be evaluated analytically. In particular, we obtain the metallic surface energy $\gamma_{s} \simeq 4 W_{c} \sqrt{2 A_{c}} / 3$ and interfacial width $\delta_{s} \sim 2 W_{c} / \sqrt{2 A_{c}}$. Therefore, the phase field model parameters $A_{c}$ and $W_{c}$ are uniquely determined from the metallic phase interface energy and width.

Within the Ginzburg-Landau formalism and with the aid of variational derivatives, the evolution of $c(\mathbf{r}, t)$ follows the conventional Cahn-Hilliard equation for conserved fields [21]. Specifically, the evolution equation is written as

$$
\frac{\partial c}{\partial t}=\nabla \cdot\left[M \nabla\left(\frac{\delta \mathcal{F}_{t o t}}{\delta c}\right)\right],
$$

where $M$ is the atomic mobility function of the metallic phase, and $\delta \mathcal{F}_{\text {tot }} / \delta c$ constitutes the driving force for the microstructural evolution. At SOFC operating temperatures, the bulk self-diffusion for metals typically used in SOFC anodes $\sim 10^{-16} \mathrm{~m}^{2} \mathrm{~s}^{-1}$ [22], whereas surface self-diffusion of these metals $D_{s} \sim 10^{-11} \mathrm{~m}^{2} \mathrm{~s}^{-1}[23]$. It is also worth noting that the $Z r^{4+}$ surface diffusion coefficient in YSZ is $\sim 10^{-18} \mathrm{~m}^{2} \mathrm{~s}^{-1}$ [24]. Therefore, it is assumed that the ceramic phase is a non-evolving one, and the evolution of the metallic one is dominated by mass transfer along metal-pore interfaces. In the present work, the atomic mobility of the metallic phase has the general form $M=$ $M_{o} Q(c, \phi, \nabla c, \nabla \phi)$, where $M_{o}$ is the atomic mobility of the metal, and the detailed form of the dimensionless function $Q$ is given in Eq. (5).

Equation (3) is made non-dimensional by introducing non-dimensional spatial coordinates $\widetilde{\mathbf{r}}=\mathbf{r} / d_{o}$ and time $\tilde{t}=t / \tau$, where $d_{o}$ and $\tau$ denote the characteristic length (voxel edge length) and time scales, respectively. For convenience, we also introduce the non-dimensional quantities $\widetilde{W}_{c}^{2}=$ $A_{c} /(2 E), \widetilde{A}_{c}=A_{c} d_{o}^{2} /\left(E \delta_{s}^{2}\right), \widetilde{\xi}_{1}=\xi_{1} /\left(E \delta_{s}^{2}\right)$, and $\widetilde{\xi}_{2}=\xi_{2} d_{o}^{2} /\left(E \delta_{s}^{2}\right)$, where $E$ is a reference energy density. Atomic mobility can be written as $M_{o}=$ 
$D_{s} /(\partial \mu / \partial c)_{c_{e q}}=D_{s} /\left(\partial^{2} f_{h o m} / \partial c^{2}\right)_{c_{e q}}$. Therefore, the characteristic time $\tau$ can be expressed as

$$
\tau=\frac{d_{o}^{4}\left(\partial^{2} f_{\text {hom }} / \partial c^{2}\right)_{c_{e q}}}{E D_{s} \delta_{s}^{2}} \simeq \frac{16 \widetilde{W}_{c}^{2} d_{o}^{4}}{D_{s} \delta_{s}^{2}} .
$$

The resulting non-dimensional evolutionary equation for $c(\mathbf{r}, t)$ has the same form as Eq. (3) and for the remainder of this paper, we drop the tildes on all parameters for notational convenience. Finally, the form used for the non-dimensional mobility function $Q$ is

$$
\begin{aligned}
Q= & a_{1}\left[1-\tanh \left(\frac{|\nabla \phi|^{2}}{\epsilon}\right)\right] \tanh \left(\frac{|\nabla c|^{2}}{\epsilon}\right)+ \\
& a_{2} \tanh \left(\frac{|\nabla \phi|^{2}}{\epsilon}\right) \tanh \left(\frac{|\nabla c|^{2}}{\epsilon}\right)+ \\
& a_{3}\left[1-\tanh \left(\frac{|\nabla c|^{2}}{\epsilon}\right)\right] \tanh \left(\frac{|\nabla \phi|^{2}}{\epsilon}\right),
\end{aligned}
$$

where the first term on the right hand side controls the mobility along metalpore interfaces, while the last two terms account for the mobility of metal along metal-ceramic and ceramic-pore interfaces, respectively. Model parameters, $a_{1}, a_{2}$ and $a_{3}$, control the contribution from each interface type, while $\epsilon$ controls the values of $c$ and $\phi$ that contribute to the mobility function.

\section{Microstructural Characterization}

In this study, the as-synthesized SOFC porous anodes are idealized as binary mixtures of metallic and ceramic spherical particles. The advantage of using this class of "virtual" microstructures is the ability to conveniently control several attributes in these systems, such as volume fraction of the constituent phases and particle sizes. These systems are then used to examine the role of metal-ceramic interaction in porous SOFC anodes and the resulting wetting angle on the evolution of several microstructural attributes affecting electrochemical performance. The random close packing (RCP) algorithm of Gan et al. [25, 26] is used to generate binary mixtures of spherical particles with periodic boundary conditions. Then, the particles are numerically sintered by expanding the radii of all spheres uniformly while keeping the center of each sphere at a fixed position, until a desired densification is reached $[27,28]$. 
In this study, RCP systems are generated such that the average volume fraction of the metal, ceramic, and pore phases correspond to $22.55 \pm 0.01 \%$, $54.11 \pm 0.02 \%$, and $23.33 \pm 0.02 \%$, respectively, similar to experimentally characterized SOFC cermet anodes $[5,6]$. The resulting virtual anode systems are digitized and mapped onto a $N_{x} \times N_{y} \times N_{z}$ uniform grid, where the $c$ and $\phi$ phase fields are assigned to the corresponding metal and ceramic particles. Next, sharp interfaces of the spherical particles are made diffuse by constructing signed distance functions from the OPs and fitting them to a hyperbolic tangent function [29]. Throughout the coarsening simulations, the phase fields $c$ and $\phi$ are used to describe the topology of the anode systems and characterize the evolving microstructures as we discuss next.

\subsection{Average Metallic Phase Diameter}

The particle size distribution (PSD) is a convenient metric typically used to analyze coarsening behavior. However, in a computational setting, the number of particles that can be simulated is often too low to generate smooth distributions. As an alternative, a ray tracing algorithm outlined in Ref. [30] is employed to track the evolution of the average phase diameter of the metallic phase particles.

In the ray tracing method, a ray is generated at each grid point within a small band around the metallic interface and is initialized to the inwardpointing, unit normal vector. The ray is propagated through the metallic phase incrementally by scaling the magnitude of the ray. When the ray reaches the opposite interface, its magnitude is recorded as a measurement of the phase diameter. The set of data generated from all of the rays is used to create ray length distributions by binning as well as determine the time-dependent average phase diameter, identified here as the average ray length.

\subsection{Three Phase Boundary Length}

Junctions in the microstructure, where the metallic, ceramic and porous phases meet, trace out the three phase boundary (TPB) lines. These indicate regions where the electrochemical reactions can potentially take place. Therefore, ensuring a sufficient number of sites for electrochemical reactions requires a large content of TPBs. However, in order for a TPB segment to actively contribute to the SOFC performance, the electrons must have a contiguous path through the anode to the interconnect via the metallic phase. 
These TPB lines are known as active, or effective, TPBs [8]. It is common practice to use the TPB density, i.e., TPB length divided by sample volume, as a simple metric to correlate the anode microstructure and the electrochemical performance of SOFCs. The methods used to calculate both the total and active TPB densities are described below.

Several computational methods have been developed to measure the amount of TPB in an anode system [19, 30]. Yet, in the current study, it is critical to ensure that the measurement of TPB length is not sensitive to changes in phase overlap, which may result from extreme wettabilities. In this work, TPB lines are extracted from the surface intersection of the metallic and ceramic level sets. To accomplish this, we employ the GNU Triangulated Surface (GTS) library [31], which is based on the algorithms detailed in Refs. [32, 33]. With the GTS library, triangulated iso-surfaces are extracted from the order parameters using level sets $c(\mathbf{r}, t)=-0.4$ and $\phi(\mathbf{r})=0.25$. Then, the boolean intersection of these two surfaces is found and represented with many small, linear segments, which denote the TPB lines. Finally, the total TPB length is calculated by summing the lengths of all the segments representing the intersection. The above level set values were chosen to ensure that the extracted surfaces overlap sufficiently for all values of metal-ceramic wetting angles used in the study.

Losses in contiguity of the electron conducting phase due to coarsening may dramatically reduce the electrochemically active TPB and performance. To account for this, the active TPB measurement is limited to TPB segments that are formed by active clusters of the metallic phase. An active cluster is defined here as one that spans the length of the system in at least one dimension. Periodic boundaries are accounted for by matching the contiguity of clusters with that of any adjacent clusters across the boundaries. Calculations of the active TPB are carried out as explained above for the total TPB, except that non-percolating metallic clusters are excluded from the analysis.

\subsection{Metallic Phase Contiguity}

Within the context of this study, contiguity is defined as the volume fraction of the metallic phase that belongs to percolating metallic clusters that span the anode system from end to end. Contiguity is an important

factor in the study of coarsening in SOFC anodes as it measures the degree of 
connectedness of the metallic phase and its ability to transport electrons from TPBs within the anode to the current collector. In this study, dimensional contiguity is defined as the volume fraction of the metallic phase that belongs to clusters that span the system along a particular dimension, or direction. Then, the reported contiguity refers to the average of the three values for the dimensional contiguity along $\mathrm{x}, \mathrm{y}$, and $\mathrm{z}$ directions.

Computationally, contiguity is calculated as follows. First, the metal phase field, $c$, is discretized into metal containing $[c(\mathbf{r}, t) \geq 0]$ and nonmetallic $[c(\mathbf{r}, t)<0]$ voxels. Then, the Hoshen-Kopelman cluster labeling algorithm [34] is utilized to identify spatially isolated metallic clusters. Each cluster is examined to determine whether it spans the system along any spatial dimension ( $\mathrm{x}, \mathrm{y}$ and/or $\mathrm{z}$ directions). The effect of periodic boundaries from the phase-field simulations is accounted for by updating the connectivity of clusters with any adjacent ones across the periodic boundaries. Finally, contiguity is calculated by averaging the dimensional contiguity along the three spatial dimensions.

\subsection{Surface Curvatures}

Local surface curvature and its probability distribution have been shown to be very useful metrics in characterizing the microstructure of complex systems $[35,36]$. Calculating the curvatures at every point along the surface of an interface and mapping these onto the plane spanned by principal curvatures gives an indication of dominant microstructural features. This mapping, which acts as a topological fingerprint of the system, offers insight into the topology that results from coarsening and the interaction between constituent phases.

Curvature maps are created at a given time during the coarsening process by calculating curvature values at interfacial patches using the order parameter field. First, the interface is smoothened by evolving the order parameter using the diffusion equation for a brief duration. Smoothing reduces the noise in the curvature data by eliminating high-frequency purturbations in the interface without significantly altering the microstructure. Then, a signed distance function is used to restrict calculations to within a narrow band of the interface. The signed distanced function is initialized from the order parameter and evolved in an iterative fashion until the value of the function at each grid point represents the distance to the closest point on the interface [37]. For the data presented in this study, the number of diffusional smoothing iterations, diffusion coefficient, and diffusion timestep are 


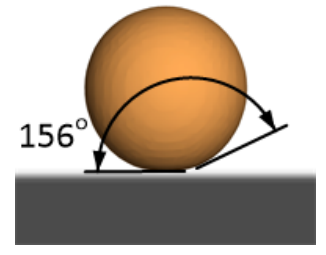

(a)

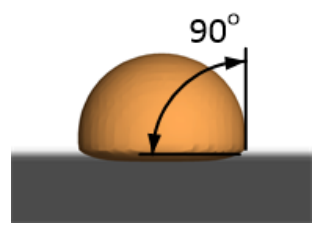

(b)

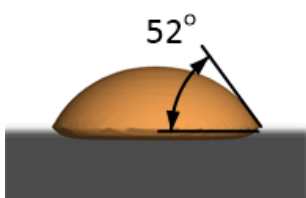

(c)

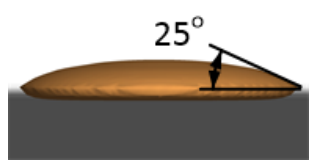

$(d)$

Figure 1: (Color online) Final configurations from simulations in which a spherical Ni droplet (orange) is placed atop a static planar YSZ substrate (grey) and equilibrates to achieve range of contact angles controlled by the model parameter $\xi_{1}$. The contact angle takes values of (a) $\theta_{c}=156^{\circ}\left(\xi_{1}=0.5\right)$, (b) $\theta_{c}=90^{\circ}\left(\xi_{1}=2.0\right)$, (c) $\theta_{c}=52^{\circ} \quad\left(\xi_{1}=3.0\right)$, (d) $\theta_{c}=25^{\circ}\left(\xi_{1}=4.0\right)$.

$(50,1.0,0.02)$. The half-width of the narrow band is $1.5 \Delta x$, where $\Delta x$ is the grid spacing.

At every gridpoint within the narrow band, the mean, $K_{M}$, and Gaussian, $K_{G}$, curvatures are calculated from the smoothened order parameter according to $[38]$

$$
\begin{aligned}
K_{M} & =\nabla \cdot\left(\frac{\nabla \phi}{|\nabla \phi|}\right), \\
K_{G} & =\frac{\nabla \phi H^{*}(\phi) \nabla \phi^{T}}{|\nabla \phi|^{4}},
\end{aligned}
$$

where $H^{*}(\phi)$ is the adjoint of the Hessian of the field $\phi$, and the superscript "T" denotes the transpose operator. Next, the principal curvatures, $\kappa_{1}$ and $\kappa_{2}$, are extracted from the mean and Gaussian ones via $K_{M}=(1 / 2)\left(\kappa_{1}+\kappa_{2}\right)$ and $K_{G}=\kappa_{1} \kappa_{2}$. Every grid point around the metallic phase interface corresponds to a point in the plane spanned by the principle curvatures. By locating peaks of the distribution in curvature space and identifying the topologies associated with the principal curvatures there, one can ascertain the dominant, microstructural features present in the system. Note that although the curvature map is somewhat sensitive to the amount of smoothing employed, the location of peaks in the distribution remain unchanged. Differing amounts of smoothing is tested to ensure the robustness of the conclusions drawn from the data. 


\section{Results}

Having outlined the computational framework and microstructural characterization techniques employed in this work, we now turn to a discussion of the results. Virtual RCP SOFC anode systems are first digitized to yield grids with $N_{x}=N_{y}=N_{z}=120$, where the voxel size is $\Delta x=\Delta y=\Delta z=1.0 d_{o}$. In dimensional units, the voxel size $d_{o} \sim 50 \mathrm{~nm}$ yields a system size $\sim 6 \mu \mathrm{m}$. To simulate systems where metallic surface diffusion is the dominant transport mechanism, the parameters that are used in the mobility function in Eq. (5) are set to $\left(a_{1}, a_{2}, a_{3}, \epsilon\right)=(1.0,0.1,0.1,0.01)$. Other parameters that are used in the simulations are $\left(A_{c}, W_{c}, \xi_{2}\right)=(0.25,1.0,0.5)$. With these model parameters, the characteristic time scale $\tau \sim 10 \mathrm{~s}$, assuming $D_{s} \sim 10^{-11} \mathrm{~m}^{2} / \mathrm{s}$ and $\delta_{s} \sim 1 \mathrm{~nm}$ in Eq. (4). The width of the ceramic-pore interface is set to $30 \%$ the metallic one. We note that the equilibrium contact angle between the metallic and ceramic phases is controlled via the $\xi_{1}$ model parameter. The evolutionary equation for the phase field, $c$, is solved using a forward-time centered-space, explicit scheme with a five-point stencil for the Laplacian operator. The time increment for the simulations is set to $\Delta t=0.002 \tau$, and data is written to disk every $4 \times 10^{5}$ timesteps. To expedite runtimes, the Message Passing Interface (MPI) protocol is used to parallelize the simulations, where three-dimensional domain decomposition is used to distribute the system in rectangular domains among the processors.

We start exploring our model by simulating sessile droplet tests in order to relate the model parameter $\xi_{1}$ to the equilibrium contact angle. To this end, we examine the evolution of an initially spherical metallic droplet on a static ceramic substrate. Panels (a)-(d) of Fig. 1 show the resulting final configurations at various values for $\xi_{1}$. The droplet lowers the system free energy by increasing the interfacial area with the substrate. Equilibrium is reached by a balance of interfacial line tensions, and the resulting contact angle is measured by visually fitting a wedge to the phase boundary, as shown in Fig. 1. As the value of $\xi_{1}$ is increased, the coupling between the metallic and ceramic phases is strengthened, and a smaller equilibrium contact angle is obtained.

Having established a correspondence between model parameters and the equilibrium contact angle, we are ready to examine the microstructural evolution of SOFC anode systems under various choices for this angle. As discussed in Sec. 3, RCP systems are used to represent porous SOFC anodes, where metallic and ceramic domains are idealized as spherical particles with 


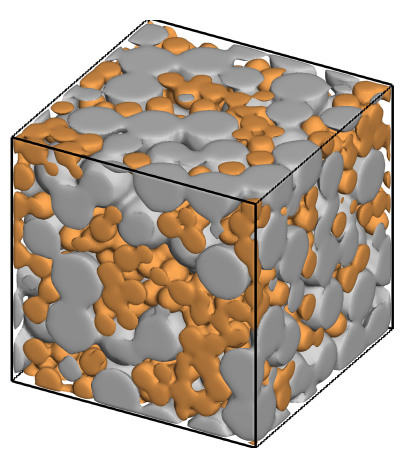

(a)

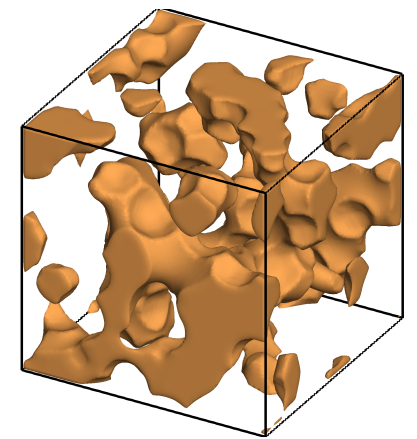

(b)

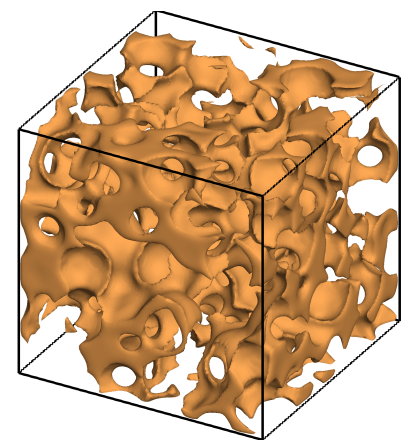

(d)

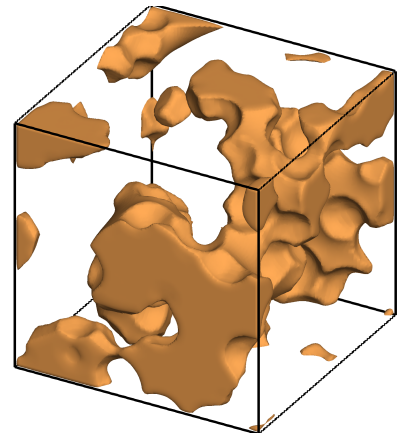

(c)

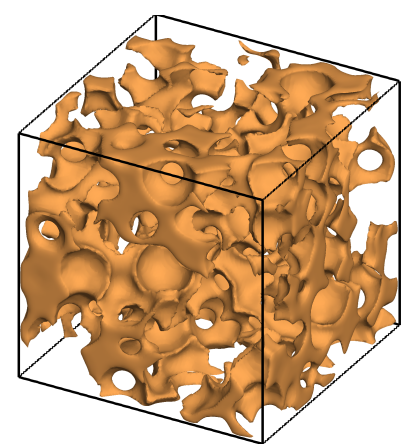

(e)

Figure 2: (Color online) Representative Ni phase configurations during the coarsening process for equilibrium wetting angles $\theta_{c}=90^{\circ}(\mathrm{a}, \mathrm{b}, \mathrm{c})$ and $\theta_{c}=25^{\circ}(\mathrm{a}, \mathrm{d}, \mathrm{e})$. The images show the configurations at $t=0$ (a), $t=8000 \tau(\mathrm{b}, \mathrm{d})$, and $t=72000 \tau(\mathrm{c}, \mathrm{e})$. The metallic phase is shaded in orange in all panels, while the non-evolving YSZ particles are shaded grey at the initial time (a) and omitted from panels (b) through (e) for visual clarity.

initial radii $12 \Delta x$ and $24 \Delta x$, respectively. Equilibrium metal-ceramic contact angles ranging from $25^{\circ}$ to $180^{\circ}$ are examined in this study. To account for sample-to-sample variations, four randomly generated initial configurations are numerically coarsened for each contact angle $\theta_{c}$, and the data is averaged over these four simulations.

The coarsening behavior can be visually inspected by monitoring the evolution of interface of the metallic phase. Figure 2 displays system configurations at initial, intermediate, and final times for both intermediate $\left(\theta_{c}=90^{\circ}\right)$ and low $\left(\theta_{c}=25^{\circ}\right)$ contact angles. It can be seen that the two representa- 
tive systems exhibit significantly different microstructures. The intermediate contact angle system is characterized by large metal clusters, while the low contact angle angle one has formed thin layers of metal around the ceramic network.

The coarsening can be examined further using the mean phase diameter, which is found using the ray tracing method described in Sec. 3. Figure 3 (a) shows the evolution of the ray length distribution for a rather poorly wetting system $\left(\theta_{c}=156^{\circ}\right)$ during the coarsening process, while Fig. 3 (b) in turn displays the time-dependent average phase diameter for eight contact angles. It can be discerned that a trend appears for small contact angles, where an increase in the contact angle results in a larger mean phase diameter at a given time. Also, the largest wettability systems $\left(\theta_{c}=25^{\circ}, 32^{\circ}\right)$ display a drop in mean phase diameter at early coarsening times, due to the interaction between metallic and ceramic phases that adjusts the contact angles in the as-synthesized microstructure. For systems simulated with an equilibrium contact angle $\theta_{c}>73^{\circ}$, on the other hand, variations in $\theta_{c}$ do not lead to significant changes in the mean phase diameter. The trend observed at lower contact angles is an indication of the "coating" type of microstructure, which is distinctly different from the microstructure of high contact angle systems. These microstructures are discussed further below.

Next, we examine the temporal evolution of TPB density as the SOFC anode systems evolve. Final configurations of the metallic phase particles are shown in Fig. 4 along with their corresponding TPB lines. As expected, the TPB lines appear along the edges of the inverted spherical caps where the ceramic particles osculate the metallic clusters. Figure 5(a) in turn shows the TPB density as a function of time for multiple contact angles. In accordance with experimental observations [8], the TPB density is a monotonically decreasing function of simulation time.

To analyze the effect of the contact angle on resulting TPB density, Fig. 5(b) shows the TPB density from all twelve equilibrium contact angles taken at early, intermediate, and late times during the coarsening process. At early and intermediate times, the TPB density monotonically decreases with increasing contact angles. As expected, systems with the highest wettability between metallic and ceramic phases, i.e., lowest contact angles, exhibit the highest TPB density. One might intuit this result by considering that increasing the shared interfacial area between the two phases geometrically lengthens their phase boundary.

At late times, on the other hand, a discernible increase in TPB density is 


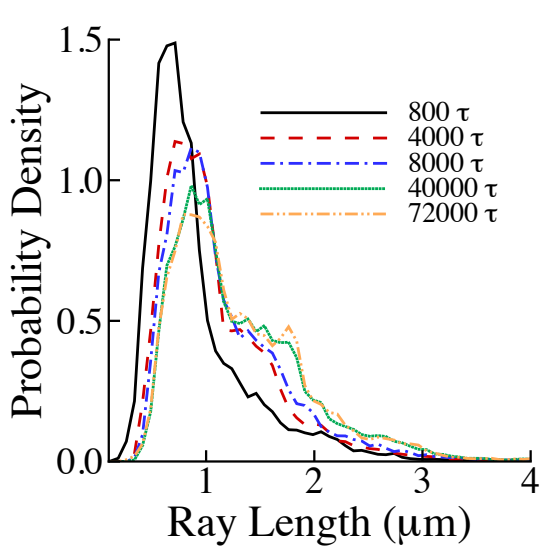

(a)

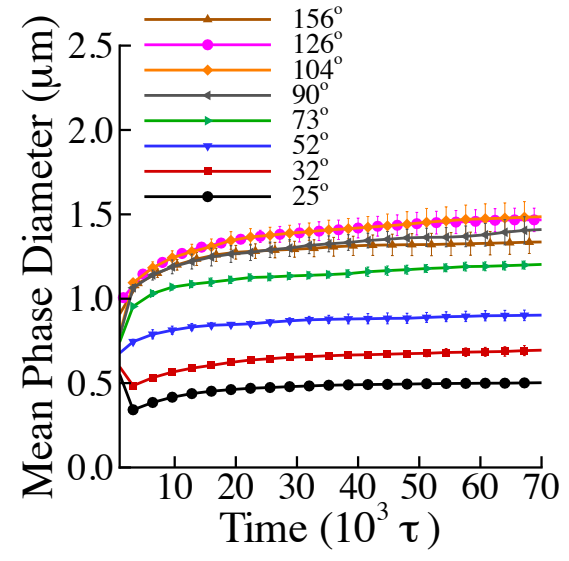

(b)

Figure 3: (Color online) (a) Metallic phase ray length distributions taken at different times for a system with equilibrium contact angle $\theta_{c}=156^{\circ}$. (b) Temporal evolution of the mean phase diameter averaged over four simulations for eight of the 12 equilibrium contact angles employed in this work.

observed for contact angles $\theta_{c} \gtrsim 130^{\circ}$. This is quite surprising, as the shared interface between $\mathrm{Ni}$ and YSZ phases is greatly reduced at large contact angles. Yet, this observation can be explained by considering the tendency of the metallic phase to wet the constricted areas between adjacent ceramic particles. As shown in Fig. 4(a), the system with contact angle $\theta_{c}=126^{\circ}$ has sufficient phase wettability that the metallic phase can wet the space between YSZ particles leaving gaps in TPB lines along those edges. On the other hand, for high contact angle systems, the phases have lower wettability and the metallic phase forms closed loops of TPB, which increases the TPB density [cf. Fig. 4(b)]. It should be noted that this increase in TPB at high contact angles arises from the spherical nature of the sintered ceramic particles and may not be observed for different ceramic particle morphologies (such as polygonal).

In contrast to the total TPB, the active TPB accounts for losses in contiguity of the electron conducting phase to give a better measurement of the electrochemically active TPB. As shown in Fig. 5(c), the active TPB displays an even stronger dependence on the contact angle. At the final stages of coarsening, multiple systems at intermediate wettability corresponding to conventional 


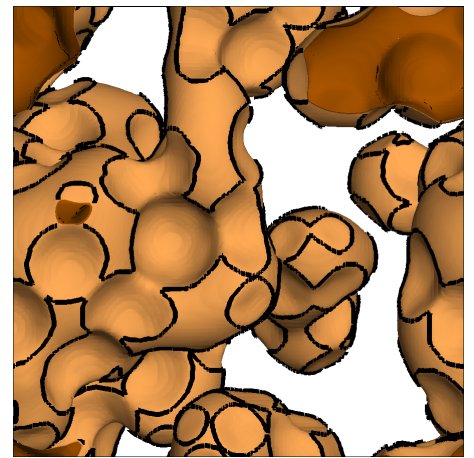

(a)

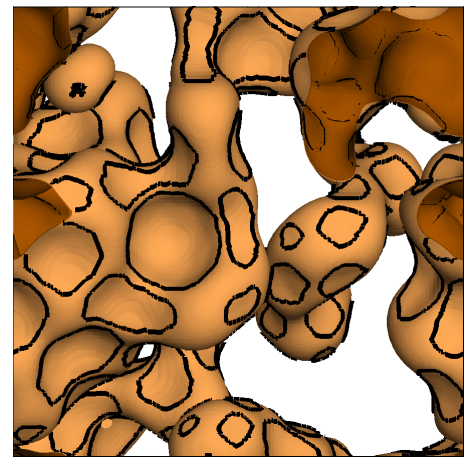

(b)

Figure 4: (Color online) Final configurations of the metallic phase particles (orange) and TPB lines (black) for systems with equilibrium contact angles of (a) $\theta_{c}=126^{\circ}$ and (b) $\theta_{c}=179^{\circ}$. Note the losses in TPB length due to merging TPB lines around the dimples formed by the YSZ particles (omitted from images for visualization purposes).

SOFC anode materials have lost all contiguity and are therefore completely depleted of active TPB. At the extremes of wettability, where systems tend to retain their contiguity (to be discussed below), nearly all of the TPB lines contribute to the density of active $\mathrm{TPB}$, as expected. We note that the rather large error bars for active TPB are primarily due to large sample-to-sample variations in contiguity.

The authors remind the reader that due to the diffuse interface nature of the model, TPB density is slightly sensitive to the method, algorithm, and cutoffs used for measurement. As a consequence, multiple methods have been employed in this study along with comparative sets of parameters to verify the robustness of the results presented in Fig. 5. The same trend is observed independent of the particularities of the data analysis with only slight differences in absolute magnitude.

We now turn our attention to contiguity, which is a metric used to measure the capability of the metallic network to transport charge across the anode. In particular, high values of contiguity correspond to well-networked metallic structures, while low values imply that electron transport processes are severly hampered. Generally, the initial metallic particles rapidly merge to form a single network that spans the length of system in every dimension. Throughout the remainder of the simulation, the nickel network may 


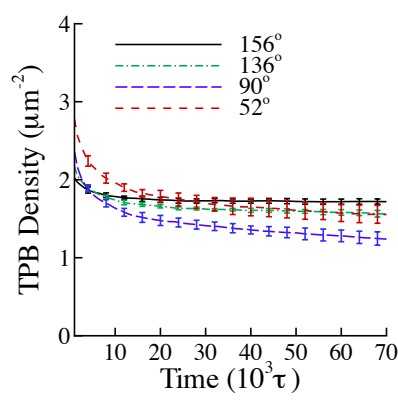

(a)

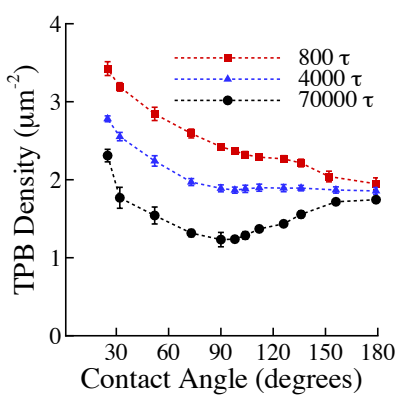

(b)

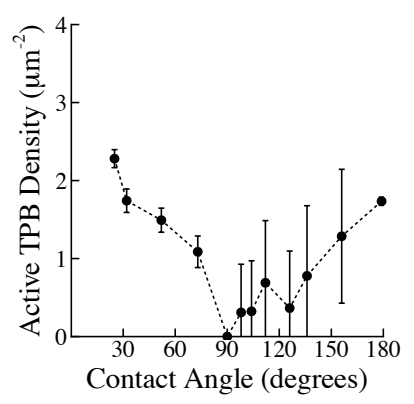

(c)

Figure 5: (Color online) (a) Temporal evolution of the total TPB density, averaged over four independent simulations, for four representative contact angles displays a monotonic decrease in TPB density over time. (b) TPB density at three specific stages (initial, intermediate, and final) during the simulated coarsening process is plotted for all contact angles employed in this work. Note the development of a minimum in TPB density at late times for $\theta_{c} \approx 90^{\circ}$. (c) Active TPB density evaluated at the final stage of coarsening displays a strong dependence on the contact angle.

split or pinch off to form smaller, isolated clusters, resulting in reduced contiguity. Of course, merging clusters can connect the metallic network and re-establish contiguous paths. These alterations in microstructure generally lead to overall losses in contiguity.

Panels (a)-(c) in Fig. 6 display contiguity data for four systems run at different contact angles. It is evident that the systems may experience dramatic losses in contiguity instantaneously. As observed through inspection, the system often reduces the total surface area by splitting the thin bridges of metal which connect two larger clusters. These pinch-off occurrences are signaled by the discrete jumps in the contiguity curves. Averaging the value of contiguity over the latter half of the simulations for each contact angle produces the graph in Fig. 6 (d). The size of the error bars are evidence of the volatile nature of contiguity losses. A system with perfect contiguity may suddenly lose all contiguity as a well-networked structure splits into two isolated clusters. The frequency of these fundamental alterations in topology and the coarsening time required to realize them vary greatly depending on the initial configuration.

These data can be rationalized as follows. At low contact angles (i.e., high wettability), it is reasonable to expect near perfect contiguity. At this 


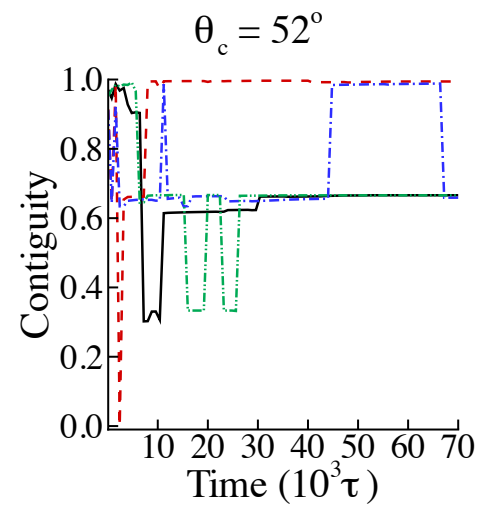

(a)

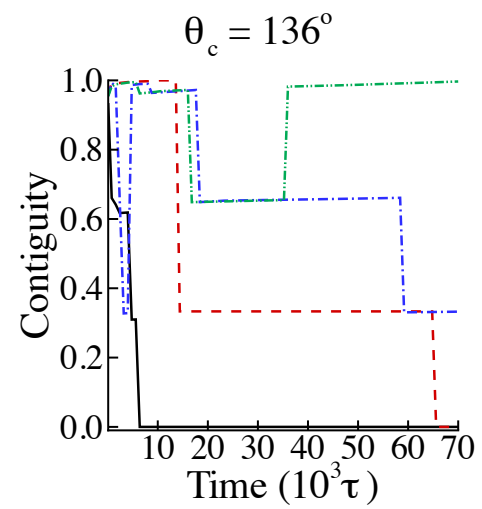

(c)

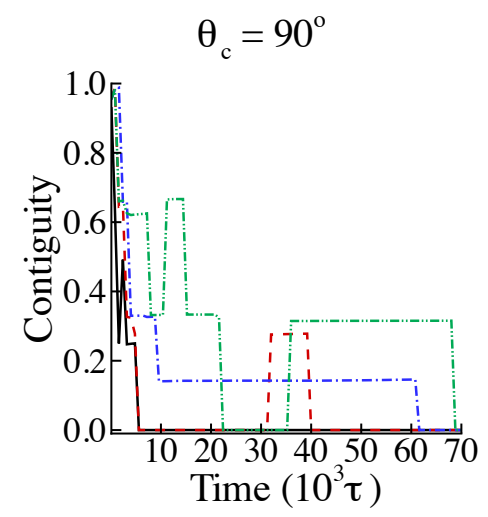

(b)

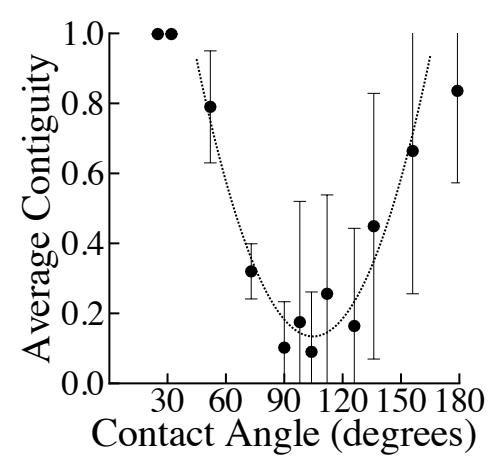

(d)

Figure 6: (Color online) Temporal evolution of contiguity for four as-synthesized RCP systems simulated with equilibrium contact angles $\theta_{c}=52^{\circ}$ [panel (a)], $\theta_{c}=90^{\circ}$ [panel (b)], and $\theta_{c}=136^{\circ}$ [panel (c)], respectively. Each individual data set was obtained by averaging the dimensional contiguities for that particular system. Next, averaging the contiguity data for the four systems over the latter half of the simulation for every contact angles produces the data shown in (d). The error bars indicate standard deviations in contiguity, and the parabolic curve is used solely to guide the eye. Note the minimum in average contiguity corresponding to $\theta_{c} \approx 100^{\circ}$.

extreme, the metallic phase interacts strongly with the ceramic network to maximize the area of shared interface. Indeed, visual inspection reveals that the metal quickly diffuses into the constricted areas between neighboring particles of the ceramic phase to form a network of concave wedges. In this 
way, the ceramic particles provide a percolating backbone on which the metal binds into a contiguous network.

At intermediate values of equilibrium contact angle, on the other hand, the systems more readily lose contiguity during the coarsening process. The interaction between the metallic and ceramic phases is not strong enough to form the percolating metallic layer microstructure that is observed at lower contact angles. Yet, in addition to coarsening, the metallic phase must also satisfy the contact angle constraint at the triple phase boundaries. We suspect that this creates a "pulling" effect, which causes the metallic network to split and hastens losses in contiguity.

Finally, the ability of systems simulated with high equilibrium contact angles (low wettability) to maintain their contiguity is somewhat surprising. Note however, the weak interaction between metal and ceramic phases in these systems allows the metal to coarsen around the ceramic backbone without the pulling effect required to satisfy lower contact angles. In order to investigate this matter further and more quantitatively, we turn to an analysis of the metallic phase topology.

Now, an analysis of surface curvature and its probability distribution yields some insight into microstructural features accompanying losses in contiguity. To this end, Fig. 7 displays final configurations of systems with low (a), intermediate (b), and high wettability (c) along with their corresponding probability densities of the principal curvatures [panels (d), (e), and (f), respectively]. In all three systems, there is a dominant microstructural feature indicated by the peak in the distribution appearing in Quadrant III of the curvature maps where $\kappa_{1}<0$ and $\kappa_{2}<0$. This peak corresponds to principal curvatures resulting from surfaces with the shape of an inverted sphere, as illustrated in Fig. 8. The presence of this peak can be attributed to the "dimpled" patches where the metallic phase shares an interface with the spherical ceramic particles.

Interestingly, there is a prominent peak in the curvature distribution of the low wettability system in Quadrant I [corresponding to $\kappa_{1}>0$ and $\kappa_{2}>0$ in panel (d)] that is absent in the higher wettability ones. The location of this peak suggests microstructural features resembling that of a wavy or slightly beaded cylinder [cf. Fig. 8]. This is confirmed by visually inspecting Fig. 7(a). These lengthy arms in the microstructure enable the system to retain its contiguity significantly longer than the system with intermdediate wettability. For the intermediate wettability system in panel (b), these spatially extended "bridges" have undergone pinch-off events due to the metal 


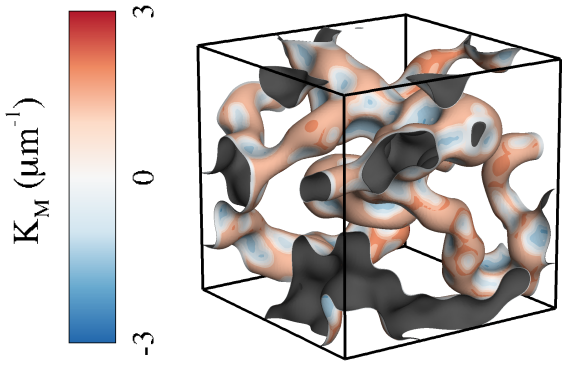

(a)

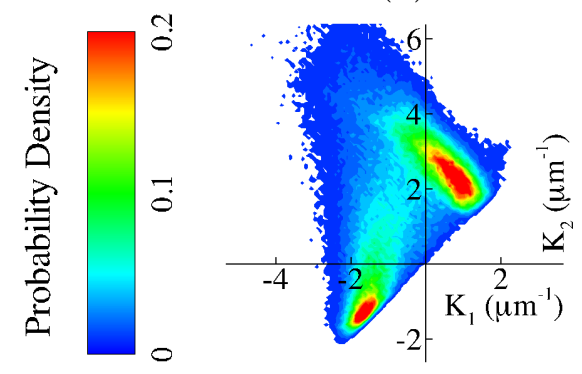

(d)

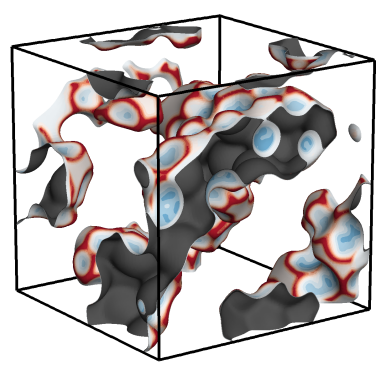

(b)

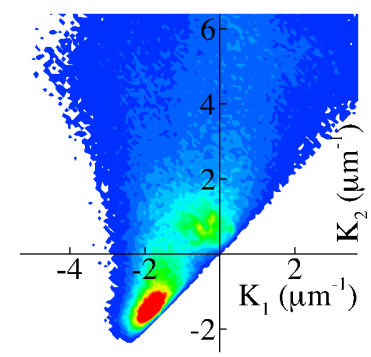

(e)

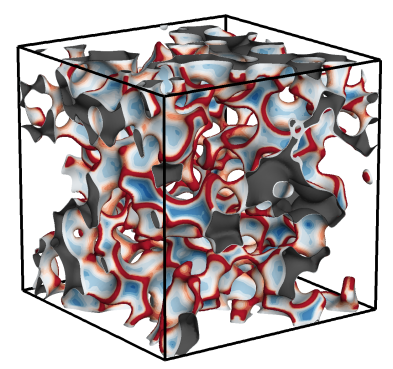

(c)

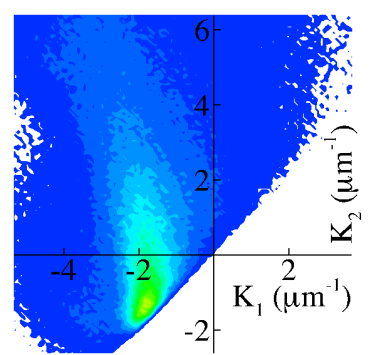

(f)

Figure 7: (Color online) Final configurations for systems with equilibrium contact angles of (a) $\theta_{c}=156^{\circ}$, (b) $\theta_{c}=90^{\circ}$, and (c) $\theta_{c}=25^{\circ}$ colored by local mean surface curvature $K_{M}$. Corresponding probability densities of the principal curvatures are shown in panels (d), (e), and (f), respectively. The location of the density peaks reveals the dominant topological shapes in the microstructure. Note that the ceramic phase particles have been omitted from the visualizations for clarity.

phase's interaction with the ceramic backbone. As a result, the metal has separated into large, disparate clusters resulting in low contiguity.

In the high wettability case, close inspection of Fig. 7(c) reveals that the metallic phase maximizes the shared interface by diffusing between neighboring ceramic particles. This results in an abundance of concave wedge shapes situated along the constricted area between the sintered particles. The outer surface of the wedges form concave menisci characterized by high, negative mean curvature, indicated by the dark blue shading. These interface patches are an additional contribution to the peak in the distribution in Fig. 7(f). This explains the broader distribution of inverted surface curvatures [corresponding to $\kappa_{1}<0$ and $\kappa_{2}<0$ ] compared to that of the lower wettability configurations. 


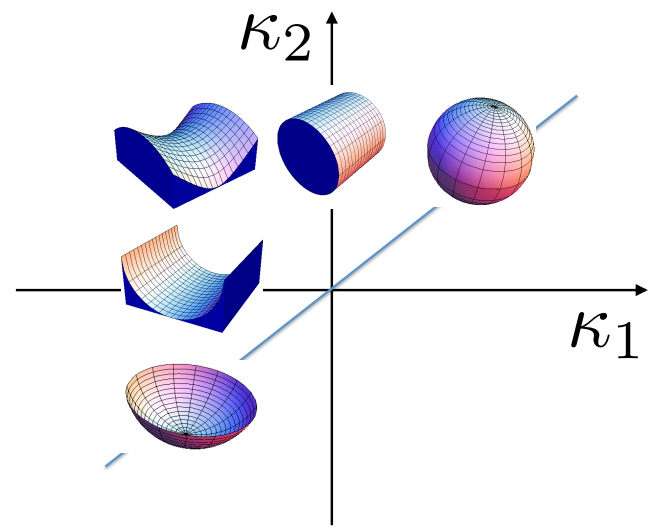

Figure 8: (Color online) A key showing the topological features associated with the various parts of the principal curvature plane to aid in identifying dominant microstructural features.

Taken together, these TPB density, contiguity, and surface curvature data strongly suggest that tuning the equilibrium contact angle between the metallic and ceramic phases can affect significant changes in performance-critical microstructural properties of SOFC anode systems. In particular, systems with low contact angles result in a metallic coating type of microstructure that has high TPB density and contiguity, resulting in good electrochemical performance. However, the data suggests that significant increases in TPB and contiguity can only be obtained at rather low contact angles $\left(\theta_{c} \lesssim 70^{\circ}\right)$.

\section{Conclusions}

Experiments have shown that the performance of solid oxide fuel cells (SOFCs) suffers from losses in three phase boundary density and contiguity, arising from coarsening processes of the metallic phase particles within the anode network $[5,6,8]$. Based on extensive simulations of such coarsening processes using a continuum diffuse-interface approach, we have shown that the wettability between the metallic network and electrolyte backbone has a significant effect on the ability of the system to retain active TPB and phase contiguity. Interestingly, both of these metrics display undesirable minima around an equilibrium contact angle of $100^{\circ}$.

Using the results from this study along with the experimental measurements, which report the Ni-YSZ equilibrium contact angle is at least $117^{\circ}[8$, 
12], we can make predictions as to the performance of SOFC anodes. As observed, materials with low contact angles result in a metallic coating type of microstructure that has high active TPB density and benefits from the high contiguity of its ceramic substrate. However, the data suggests that significant increases in TPB and contiguity can only be obtained at very low contact angles, less than $70^{\circ}$. This is a rather extreme alteration in material properties. On the other hand, performance enhancement may also be gained by an increase in the equilibrium contact angle. At this extreme, curvature maps indicate that cylindrical bridges retain the system contiguity, while active TPB density is slightly larger due to the separation between metallic-ceramic interface patches.

Further model refinements are desirable for continued study of the effect of wettability on metallic coarsening. Abdeljawad et al. [7] have shown using a polycrystalline model for the metallic phase that perturbations induced by thermal grooving assist in the growth of Rayleigh-type instabilities. This effect, along with excess grain boundary energy and a potential interface mobility at grain boundary "pinch-off" sites, may affect the ability of the system to retain contiguity. The challenge is generalizing the model to enable multiple grain orientations while limiting computational complexity to allow for simulation of long-time-scale degradation mechanisms. Additional inquiries involve additional volume fractions and particle ratios. We hope to report on these issues in a future publication.

\section{ACKNOWLEDGMENTS}

This work has been supported by the Energy Frontier Research Center on Science Based Nano-Structure Design and Synthesis of Heterogeneous Functional Materials for Energy Systems funded by the U.S. Department of Energy, Office of Science, Office of Basic Energy Sciences (award DE-SC0001061), and by the NSF-REU "EMERGE" program at Princeton University (award DMR-1156422). We would also like to thank Dr. Ben-

jamin Völker and Professor Robert M. McMeeking for providing the RCP microstructures employed as initial conditions in this work.

\section{References}

[1] Atkinson, A., Barnett, S., Gorte, R.J., Irvine, J.T.S., McEvoy, A.J., Mogensen, M., et al. Nat Mater 2004;3(1):17-27. 
[2] Choudhury, A., Chandra, H., Arora, A.. Renew Sustain Energy Rev 2013;20:430-442.

[3] Stambouli, A., Traversa, E.. Renew Sustain Energy Rev 2002;6(5):433455.

[4] Ryan, E., Xu, W., Sun, X., Khaleel, M.. J Power Sources 2012;210:233-242.

[5] Tanasini, P., Cannarozzo, M., Costamagna, P., Faes, A., Van Herle, J., Hessler-Wyser, A., et al. Fuel Cells 2009;9(5):740-752.

[6] Faes, A., Hessler-Wyser, A., Presvytes, D., Vayenas, C.G., Van herle, J.. Fuel Cells 2009;9(6):841-851.

[7] Abdeljawad, F., Völker, B., Davis, R., McMeeking, R.M., Haataja, M.. J Power Sources 2014;250:319-331.

[8] Nelson, G.J., Grew, K.N., Izzo, J.R., Lombardo, J.J., Harris, W.M., Faes, A., et al. Acta Mater 2012;60(8):3491-3500.

[9] Gorte, R., Vohs, J.. Curr Opin Colloid Interface Sci 2009;14(4):236244.

[10] Grgicak, C.M., Pakulska, M.M., OBrien, J.S., Giorgi, J.B.. J Power Sources 2008;183(1):26-33.

[11] Hornés, A., Gamarra, D., Munuera, G., Conesa, J., Martínez-Arias, A.. J Power Sources 2007;169(1):9-16.

[12] Tsoga, A., Naoumidis, A., Nikolopoulos, P.. Acta Mater 1996;44(9):3679-3692.

[13] Tsoga, A., Nikolopoulos, P., Naoumidis, A.. Ionics (Kiel) 1996;2(56):427-434.

[14] Ringuedé, A., Bronine, D., Frade, J.. Electrochim Acta 2002;48(4):437-442.

[15] Skarmoutsos, D., Nikolopoulos, P., Tsoga, A.. Ionics (Kiel) 1999;5(5$6): 455-459$. 
[16] Provatas, N., Elder, K.. Phase-Field Methods in Materials Science and Engineering. 1 ed.; Wiley-VCH; 2010.

[17] Wilson, J.R., Kobsiriphat, W., Mendoza, R., Chen, H.Y., Hiller, J.M., Miller, D.J., et al. Nat Mater 2006;5(7):541-4.

[18] Chen, H.Y., Yu, H.C., Scott Cronin, J., Wilson, J.R., Barnett, S.A., Thornton, K.. J Power Sources 2011;196(3):1333-1337.

[19] Kim, J.H., Liu, W.K., Lee, C.. Comput Mech 2009;44(5):683-703.

[20] Li, Q., Liang, L., Gerdes, K., Chen, L.Q.. Appl Phys Lett 2012;101(3):033909.

[21] Cahn, J.W., Hilliard, J.E.. J Chem Phys 1958;28(2):258.

[22] Maier, K., Mehrer, H., Lessmann, E., Schüle, W.. Phys status solidi 1976;78(2):689-698.

[23] Bonzel, H., Latta, E.. Surf Sci 1978;76(2):275-295.

[24] Akash, , Mayo, M.. J Mater Sci 2000;5(1):437-442.

[25] Gan, Y., Kamlah, M.. J Mech Phys Solids 2010;58(2):129-144.

[26] Gan, Y., Kamlah, M., Reimann, J.. Fusion Eng Des 2010;85(1012):1782-1787.

[27] Völker, B., McMeeking, R.M.. J Power Sources 2012;215:199-215.

[28] Völker, B., McMeeking, R.M.. Effect of pore former particles on effective properties and performance in solid oxide fuel cell electrodes; 2013. Submitted to J. Power Sources.

[29] Yu, H.C., Chen, H.Y., Thornton, K.. Model Simul Mater Sci Eng 2012;20(7):075008.

[30] Grew, K.N., Peracchio, A.A., Chiu, W.K.. J Power Sources 2010;195(24):7943-7958.

[31] Popinet, S.. GTS: GNU Triangulated Surface library. http://gts.sourceforge.net/; 2000-2004. 
[32] Krishnan, S., Manocha, D.. ACM Trans Graph 1997;16(1):74-106.

[33] Barequet, G., Chazelle, B., Guibas, L.J., Mitchell, J.S., Tal, A.. Comput Graph Forum 1996;15(3):387-396.

[34] Hoshen, J., Kopelman, R.. Phys Rev B 1976;14(8):3438-3445.

[35] Mendoza, R., Savin, I., Thornton, K., Voorhees, P.W.. Nat Mater $2004 ; 3(6): 385-8$.

[36] Kammer, D., Voorhees, P.. Acta Mater 2006;54(6):1549-1558.

[37] Osher, S., Fedkiw, R.. Level Set Methods and Dynamic Implicit Surfaces. Springer; 2003.

[38] Goldman, R.. Comput Aided Geom Des 2005;22(7):632-658. 
Figure 1: (Color online) Final configurations from simulations in which a spherical Ni droplet (orange) is placed atop a static planar YSZ substrate (grey) and equilibrates to achieve range of contact angles controlled by the model parameter $\xi_{1}$. The contact angle takes values of (a) $\theta_{c}=156^{\circ}$ $\left(\xi_{1}=0.5\right),(\mathrm{b}) \theta_{c}=90^{\circ}\left(\xi_{1}=2.0\right),(\mathrm{c}) \theta_{c}=52^{\circ}\left(\xi_{1}=3.0\right),(\mathrm{d}) \theta_{c}=25^{\circ}$ $\left(\xi_{1}=4.0\right)$.

Figure 2: (Color online) Representative $\mathrm{Ni}$ phase configurations during the coarsening process for equilibrium wetting angles $\theta_{c}=90^{\circ}$ (a, b, c) and $\theta_{c}=25^{\circ}(\mathrm{a}, \mathrm{d}, \mathrm{e})$. The images show the configurations at $t=0$ (a), $t=8000 \tau$ $(\mathrm{b}, \mathrm{d})$, and $t=72000 \tau(\mathrm{c}, \mathrm{e})$. The metallic phase is shaded in orange in all panels, while the non-evolving YSZ particles are shaded grey at the initial time (a) and omitted from panels (b) through (e) for visual clarity.

Figure 3: (Color online) (a) Metallic phase ray length distributions taken at different times for a system with equilibrium contact angle $\theta_{c}=156^{\circ}$. (b) Temporal evolution of the mean phase diameter averaged over four simulations for eight of the 12 equilibrium contact angles employed in this work.

Figure 4: (Color online) Final configurations of the metallic phase particles (orange) and TPB lines (black) for systems with equilibrium contact angles of (a) $\theta_{c}=126^{\circ}$ and (b) $\theta_{c}=179^{\circ}$. Note the losses in TPB length due to merging TPB lines around the dimples formed by the YSZ particles (omitted from images for visualization purposes).

Figure 5: (Color online) (a) Temporal evolution of the total TPB density, averaged over four independent simulations, for four representative contact angles displays a monotonic decrease in TPB density over time. (b) TPB density at three specific stages (initial, intermediate, and final) during the simulated coarsening process is plotted for all contact angles employed in this work. Note the development of a minimum in TPB density at late times for $\theta_{c} \approx 90^{\circ}$. (c) Active TPB density evaluated at the final stage of coarsening displays a strong dependence on the contact angle.

Figure 6: (Color online) Temporal evolution of contiguity for four as-synthesized RCP systems simulated with equilibrium contact angles $\theta_{c}=52^{\circ}$ [panel (a)], $\theta_{c}=90^{\circ}$ [panel (b)], and $\theta_{c}=136^{\circ}$ [panel (c)], respectively. Each individual data set was obtained by averaging the dimensional contiguities for that 
particular system. Next, averaging the contiguity data for the four systems over the latter half of the simulation for every contact angles produces the data shown in (d). The error bars indicate standard deviations in contiguity, and the parabolic curve is used solely to guide the eye. Note the minimum in average contiguity corresponding to $\theta_{c} \approx 100^{\circ}$.

Figure 7: (Color online) Final configurations for systems with equilibrium contact angles of (a) $\theta_{c}=156^{\circ}$, (b) $\theta_{c}=90^{\circ}$, and (c) $\theta_{c}=25^{\circ}$ colored by local mean surface curvature $K_{M}$. Corresponding probability densities of the principal curvatures are shown in panels (d), (e), and (f), respectively. The location of the density peaks reveals the dominant topological shapes in the microstructure. Note that the ceramic phase particles have been omitted from the visualizations for clarity.

Figure 8: (Color online) A key showing the topological features associated with the various parts of the principal curvature plane to aid in identifying dominant microstructural features. 